\title{
m6A modification in RNA: biogenesis, functions and roles in gliomas
}

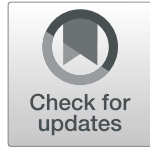

\author{
Yuhao Zhang ${ }^{1 \dagger}$, Xiuchao Geng ${ }^{2 \dagger}$, Qiang $\mathrm{Li}^{3}$, Jianglong Xu', Yanli Tan ${ }^{4}$, Menglin Xiao ${ }^{1}$, Jia Song ${ }^{5}$, Fulin Liu ${ }^{6 *}$, \\ Chuan Fang ${ }^{1 *}$ and Hong Wang ${ }^{1,2,7^{*}}$
}

\begin{abstract}
The chemical modification of RNA is a newly discovered epigenetic regulation mechanism in cells and plays a crucial role in a variety of biological processes. N6-methyladenine (m6A) mRNA modification is the most abundant form of posttranscriptional RNA modification in eukaryotes. Through the development of m6A RNA sequencing, the relevant molecular mechanism of m6A modification has gradually been revealed. It has been found that the effect of m6A modification on RNA metabolism involves processing, nuclear export, translation and even decay. As the most common malignant tumour of the central nervous system, gliomas (especially glioblastoma) have a very poor prognosis, and treatment efficacy is not ideal even with the application of high-intensity treatment measures of surgery combined with chemoradiotherapy. Exploring the origin and development mechanisms of tumour cells from the perspective of tumour biogenesis has always been a hotspot in the field of glioma research. Emerging evidence suggests that m6A modification can play a key role in gliomas through a variety of mechanisms, providing more possibilities for early diagnosis and targeted therapy of gliomas. The aim of the present review is to focus on the research progress regarding the association between m6 A modification and gliomas. And to provide a theoretical basis according to the currently available literature for further exploring this association. This review may provide new insights for the molecular mechanism, early diagnosis, histologic grading, targeted therapy and prognostic evaluation of gliomas.
\end{abstract}

Keywords: m6A modification, RNA, Central nervous system, Glioma, Glioblastoma, GBM, Tumourigenesis

\section{Background}

Gliomas are the most common malignancy in the central nervous system. Glioblastoma (GBM) has the highest malignancy rate and account for $50 \%$ of all brain tumours. The average survival time of patients with GBM is only 14.6 months [1]. GBM originate from poorly differentiated glial cells and have the characteristics of nuclear atypia, cellular polymorphism, and a high

\footnotetext{
* Correspondence: Iful666@sina.com; chuanfang@hbu.edu.cn; bossw@vip.sina.com

'Yuhao Zhang and Xiuchao Geng contributed equally to this work.

${ }^{6}$ Office of Academic Research, Affiliated Hospital of Hebei University, 071000 Baoding, China

'Department of Neurosurgery, Affiliated Hospital of Hebei University, 071000 Baoding, China

Full list of author information is available at the end of the article
}

degree of mitotic activity. Given the aggressiveness of GBM, surgical resection to resolve all intracranial lesions in clinical practice is challenging. Therefore, most patients receive radiotherapy and temozolomide (TMZ) combined with chemotherapy after surgery. Although the treatment intensity is very high, the outcomes are still not satisfactory [2-5]. Therefore, there is an urgent need for new treatment strategies or regimens.

To improve the efficacy of GBM treatment, it is necessary to understand the occurrence and development of GBM and determine the molecular biological characteristics of GBM. In recent years, with the in-depth study of the epigenetics, metabolism, and immunology of GBM, our knowledge of GBM has greatly expanded, which provides new clues for the treatment of GBM. 
Recent studies have found that non-coding RNAs and post transcriptional modification of RNAs have become the active fields of cancer research. Among them, N6methyladenine (m6A) RNA modification is the important research hotspot [6,7]. More than $60 \%$ of all RNA modifications are methylated modifications, and m6A is the most abundant chemical modification in eukaryotic messenger RNA (mRNA). As a representative of relevant studies, epigenetics found that m6A RNA modification plays important roles in the regulation of cell fate, proliferation, and metabolism and the biogenesis of tumours [6-9]. It also opens a new way of thinking for biomedical scientists, whether RNA modification will be another important factor to regulate the biological development of neoplastic diseases.

In this review, we provide a comprehensive introduction of the latest research progress on m6A modification and elucidate the origin of m6A modification, its regulation, biological functions and its correlation with the central nervous system and gliomas. And to discuss the prospect of the possible research directions. Aiming at providing a theoretical basis according to the currently available literature for further exploring the association between m6A modification and gliomas.

\section{The molecular mechanisms of m6A modification Origin of $\mathrm{m} 6 \mathrm{~A}$ modification}

With the advent of high-throughput sequencing technology, scientists have identified nearly 170 types of RNA modification, also known as "epitranscriptomics" [10]. Inserting information beyond the information carried by their base sequences to gene transcripts, altering the charge of RNA bases and their matching properties, differential folding of RNAs, and the formation of a regulatory protein-RNA interaction recognition element are classical transcriptome modification processes. Therefore, these modifications are involved in the whole process of RNA metabolism, regulating the fine expression patterns of genes and, ultimately, affecting the biological behaviour of cells $[11,12]$.

When the m6A modification was first discovered in mRNA, only a few modified sites were mapped in viral and cellular RNAs, but not in the human coding region or noncoding region [13]. In 2012, Dominissini and colleagues developed m6A sequencing (m6A-seq) technology. They presented the first modification landscape in a transcriptome-wide manner using $\mathrm{m} 6 \mathrm{~A}$-seq, proved the evolutionary conservation of $\mathrm{m} 6 \mathrm{~A}$ sites and identified dynamically modulated sites in responses to stimuli in cells. Gene sequence analysis showed that in the consensus sequence RRACH (in which R represents A or G and $\mathrm{H}$ represents $\mathrm{A}, \mathrm{C}$ or $\mathrm{U}$ ), m6A modification usually occurs [14].

\section{Approaches of detecting of $\mathrm{m} 6 \mathrm{~A}$ modification}

The total amount of m6A in RNA can be probed by several approaches, including two-dimensional thin layer chromatography, m6A dot-blot and high-performance liquid chromatography-tandem mass spectrometry (HPLCMS/MS) [15, 16].

The transcriptome-wide distribution of m6A was unclear before 2012, until methylated RNA immunoprecipitation followed by high-throughput sequencing (MeRIPseq or m6A-seq) was developed $[17,18]$. In this method, mRNA was fragmented into 100-nt-long oligonucleotides and immunoprecipitated with a specific antibody against m6A. Then immunoprecipitated RNAs were subjected to high-throughput sequencing. Other approaches for higher resolution, such as photo-crosslinking-assisted m6A-sequencing (PA-m6A-Seq) and site-specific cleavage and radioactive-labelling followed by ligation-assisted extraction and thin-layer chromatography (SCARLET) $[19,20]$. Currently, a novel method called m6A individual nucleotide resolution crosslinking immunoprecipitation (miCLIP) has marked a major step forward in the field, which could detect $\mathrm{m} 6 \mathrm{~A}$ at precise position [21]. Furthermore, with the development of CRISPR-based genome engineering, it is currently possible to directly detect the effect of altering any m6A modification site in many organisms. As a supplementary approach, it would be valuable for discovering the functions of m6A methylation $[22,23]$.

Current studies have shown that in addition to mRNA, microRNA (miRNA), long noncoding RNAs (lncRNA), circular RNA (circRNA), ribosomal RNA (rRNA), transfer RNA (tRNA) and small nucleolar RNAs (snoRNA) all have m6A modifications and that their regulation involves almost all categories of protein-coding genes and noncoding genes [24, 25].

\section{Regulation of m6A modification}

m6A modification mainly occurs in adenines in the RRACH sequence, and its functions are implemented by RNA methyltransferases (writers), RNA demethylase (erasers) and m6A binding protein (readers). We summarized the types of proteins involved in $\mathrm{m} 6 \mathrm{~A}$ modification (Fig. 1). In addition, we offered some explanations for some of these important biological functions.

\section{m6A "writer"}

Methyltransferase like 3 (METTL3) is a key protein with molecular weight of $70 \mathrm{kDa}$ and was the first protein identified as an "m6A writer" [26-28]. Gel filtration chromatography shows that METTL3 and METTL14 can form a stable and asymmetric METTL3-14 complex with a stoichiometric ratio of $1: 1$; then, this complex combines with Wilms' tumour 1-associating protein (WTAP) to play a methylation function [27, 29]. In 


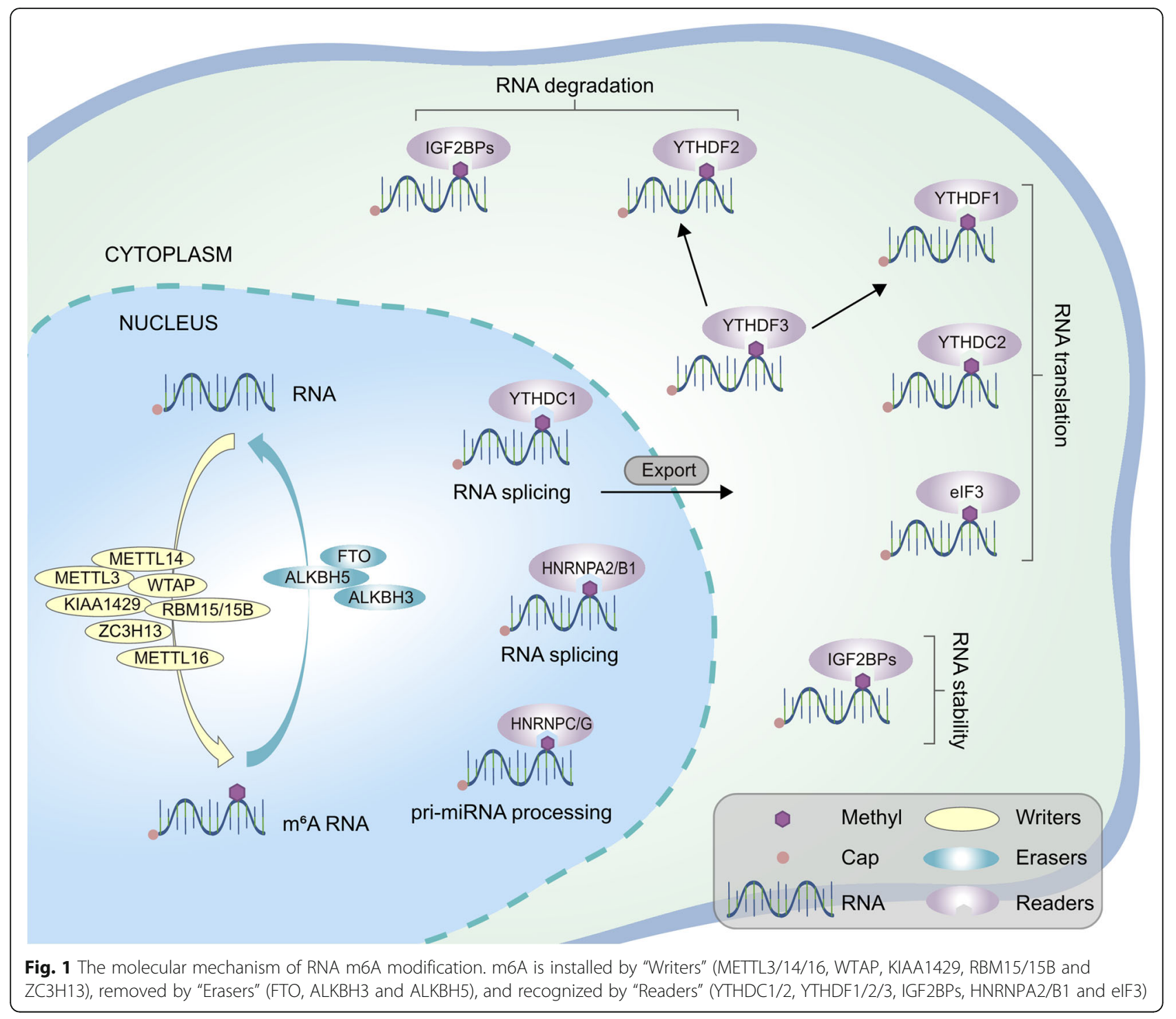

addition, METTL3 can play a central catalytic role towards methyl groups, allowing these groups to partially transfer from the S-adenosylmethionine (SAM) moiety to the receptor adenine. At the same time, METTL14 plays an important role in promoting substrate binding [30]. WTAP interacts with the METTL3-14 complex to affect m6A methyltransferase activity and localization in nuclear speckles [26, 27].

\section{m6A "eraser"}

After fat mass and obesity-associated gene (FTO) knockdown, the m6A level in mRNA increases [31]. Meyer C et al. noted that FTO has a high affinity for m6A modifications; because $\mathrm{m} 6 \mathrm{~A}$ is a reversible RNA modification, FTO can affect the fate of cellular mRNA. In this process, FTO preferentially demethylates $\mathrm{m} 6 \mathrm{~A}$ and reduces the stability of the m6A-related mRNA [32]. AlkB homologue 5, RNA demethylase (ALKBH5) resides in the nucleus, and the m6A level in mRNA is significantly reduced in cells that overexpress ALKBH5. ALKBH5's depletion affects mRNA export and assembly processes [33].

\section{m6A "reader"}

YTH N6-methyladenosine RNA binding protein 1 (YTHD F1) promotes the translation of m6A-modified mRNA, YTHDF2 accelerates RNA decay, and YTHDF1/2 and YTHDF3 synergistically promote RNA metabolism in the cytoplasm [34-36]. In the localization of nuclear speckles, under the action of YTH domain containing 1 (YTHDC1), serine-rich splicing factor 3 (SRSF3) expression is promoted, and SRSF10 expression is inhibited, thereby regulating the splicing of mRNA [37]. In addition, YTHDC2 preferentially binds to m6A-containing transcripts, thereby reducing mRNA abundance and improving translation efficiency through interactions with translation initiation and decay mechanisms [38]. 


\section{Biological functions of $\mathbf{m 6 A}$ modification m6A modification involved in RNA metabolism}

m6A modification is involved in the regulation of almost all processes of RNA metabolism. For mRNA, m6A is involved in the regulation of the processing and expression of pre-mRNA (precursor RNA) in the nucleus and the translation and decay of mature mRNA in the cytoplasm (Fig. 2) [39]. The regulation of the alternative splicing of precursor mRNA [35] is achieved through the activation of the SRSF3 pathway by the binding of m6A to YTHDC1 in the nucleus, which mainly involves the exons at the ends of mRNA. This process is also the regulatory mechanism for the diversity of adenosine polymer [40, 41]. METTL3, ALKBH5, and YTHDC1 also play important roles in the regulation of mRNA nuclear export [33, 37]. In addition, the diverse mechanism of translation regulation is a major function of $\mathrm{m} 6 \mathrm{~A}$ in the cytoplasm. m6A can not only regulate the translation efficiency of mRNA through the YTHDF1-eIF3 pathway [34] but can also mediate related processes through insulin-like growth factor-II mRNA binding protein (IGF2BP) [42]. During cellular stress responses, signalling pathways regulating 5'-end cap structure independent translation is also dependent on m6A [43]. Second, m6A also plays an important role in maintaining mRNA stability [42], of which the mechanism mainly involves the recruitment of mRNA into the processing bodies ( $\mathrm{P}$ body) through YTHDF2, followed by biological degradation [44]. Liu et al. also found that m6A can fully participate in the entire RNA metabolism process through the regulation of the RNA secondary structure; furthermore, this function of m6A modification is also closely related to the biogenesis and development of neoplastic diseases $[45,46]$.

m6A modification regulates the directed differentiation of haematopoietic stem cells

METTL3-mediated m6A modification is involved in the regulation of multiple biological functions in eukaryotic organisms. In recent years, relevant studies have found

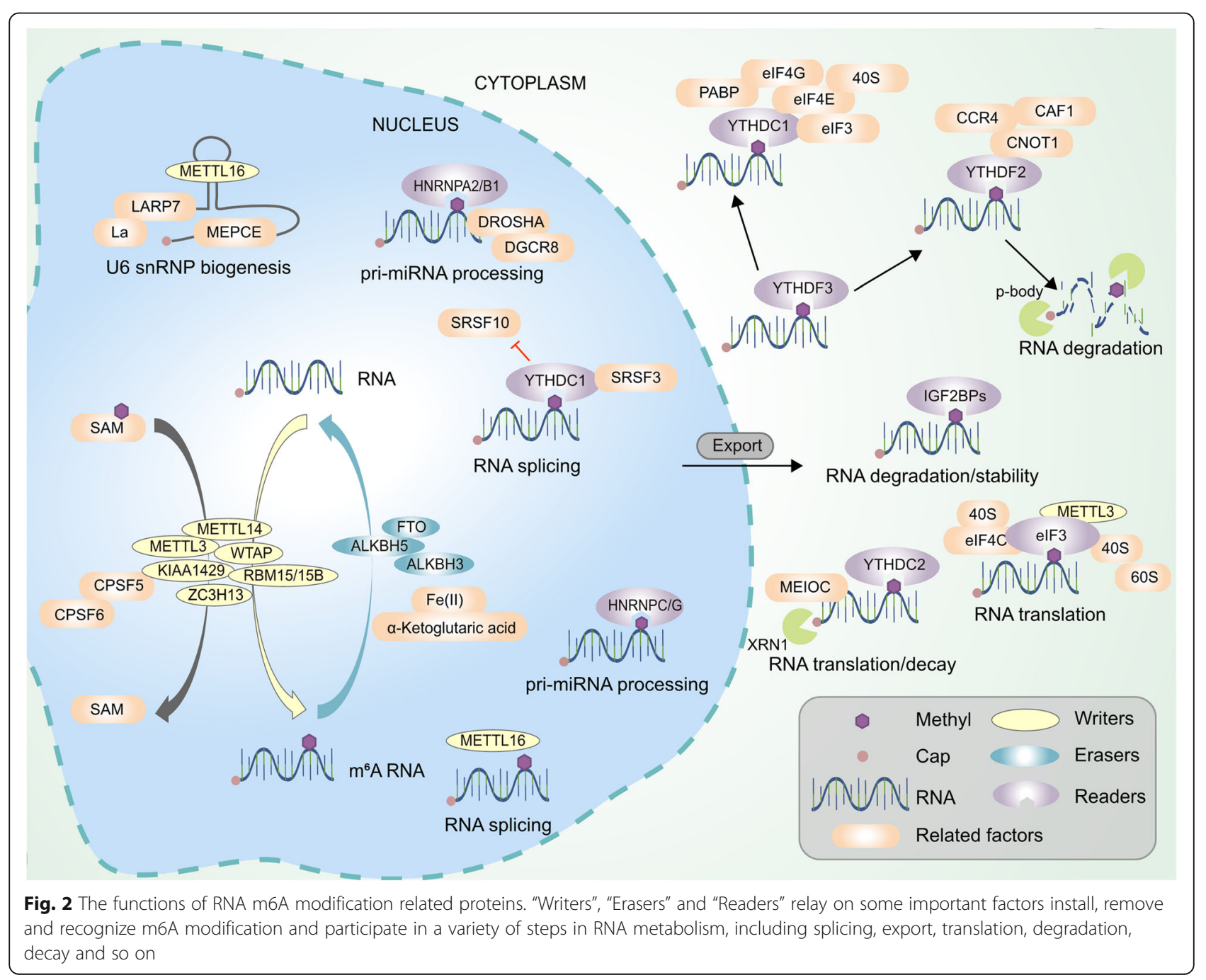


that it is closely related to biological rhythm, stem cell self-renewal, maternal-to-zygotic transition, DNA damage response, neurological function regulation and sex determination in Drosophila and early mouse embryonic development [47-56].

Zhang et al. completed the m6A modification profile for zebrafish embryogenesis and found that in the process of EHT, as a key factor, neurogenic locus Notch homologue protein 1 (Notch1a) under m6A-specific modification initiates the binding of YTHDF2 and mRNA decay, resulting in the inhibition of the Notch pathway, thereby allowing the programmed occurrence of aforementioned biological behaviours and ultimately endothelial cell transition into haematopoietic stem cells (HSPCs) [57]. In addition, after METTL3-specific knockout in mouse embryos, similar functional phenotypes were observed [58]. In general, m6A modification plays an important regulatory role in the process of vertebrate HSPC differentiation and even in the blood development process.

\section{m6A modification and the maintenance of embryonic stem cell (ESC) pluripotency}

Epigenetic and epitranscriptomic networks play important roles in somatic cell reprogramming and in the maintenance of ESC pluripotency. Recent studies have shown that zinc finger protein 217 (ZFP217) activates the transcription of key pluripotency genes and regulates m6A deposition on transcripts. Zfp217 depletion generally enhances the m6A modification of Nanog, sex determining region $\mathrm{Y}$ box 2 (Sox2), Kruppel-like factor 4 (Klf4) and c-Myc mRNAs, thereby accelerating their decay and directly destroying ESC self-renewal and somatic cell reprogramming [51, 52]. This finding reveals the close relationship between $\mathrm{m} 6 \mathrm{~A}$ and somatic cell reprogramming and the maintenance of ESC pluripotency.

m6A modification and the regulation of spermatogenesis Geula et al. found that METTL3-specific knockout in mice leads to early embryonic lethality [52]. Using the CRISPR/Cas9 and Cre-loxP systems, Xu et al. constructed a mouse model based on homologous recombination technology, specifically the knockdown of METTL3 in germ cells (Vasa-Cre). Haematoxylin-eosin (HE) staining and immunofluorescence staining showed that the differentiation and meiosis of spermatogonial stem cells in male mice were significantly inhibited, leading to infertility. In addition, the researchers also found that after METTL3 knockout, not only was the m6A RNA modification level reduced but also altered RNA alternative splicing and expression profiles were observed in the genes functioning in the maintenance of spermatogonial stem cell differentiation and cell meiosis, seriously affecting the biological formation of gametes [59].
In addition, using Vasa-Cre technology, Lin et al. specifically knocked out METTL14 in the germ cells of mice, significant disruption of the translation function of transcription products of spermatogonial stem cell proliferation and differentiation was also observed, eventually leading to the depletion of spermatogonial stem cells. Stra8-GFP Cre technology was used for the simultaneous knockout of METTL3 and METTL14, the coding and translation of haploid-specific genes was incorrect, and sperm production was inhibited [60]. On the other hand, animal experiments showed that model mice presented testicular shrinkage, spermatogenesis abnormalities, and poor sperm motility after ALKBH5 overexpression [33]. After YTHDC2 knockout, apoptosis occurred in spermatogonial cells at the early stage of meiosis, resulting in testicular shrinkage and spermatogenesis disorder [36, 61]. In summary, maintaining m6A modification homeostasis is critical for the regulation and protection of normal sperm development.

\section{m6A modification and the regulation of brain development}

Animal experiments showed that the abundance of m6A was significantly higher in the cerebellum than in the cerebral cortex of mice, suggesting that m6A may play a critical role in this anatomical region [62]. Wang et al. studied the role of m6A modification in the development of the central nervous system. Scientists used the Cre-loxP system to silent the METTL3 gene in the brains of mice. The study found that the model mice had severe movement disorders during the lactation period; some mice even died. Morphological observation of dead mice revealed severe dysplasia in both the cerebral cortex and cerebellar area. Mice in which the METTL3 gene was silenced showed severe cerebellar hypoplasia, which might be associated with the rapid apoptosis of cerebellar granule cells (CGCs) in the external granular layer (EGL) of new-born mice [63].

Studies have shown that the dynamic process of m6A modification occurs throughout the entire process of cerebellum development after a mouse is born. In the case of hypobaric hypoxia, the presence of ALKBH5 gene deletion directly lead to altered m6A levels during cerebellar development and accelerated RNA nuclear export, seriously affecting the developmental process of the cerebellum [64]. In addition, after METTL14-specific knockout, the development of the mouse cerebral cortex was also severely impaired [65]. Li et al. found that the deletion of the YTHDF2 gene in mice not only improved the level of m6A modification but also inhibited the normal RNA decay of the genes and proteins involved in neural stem cell differentiation and neuron dendrite formation; therefore, neural stem cells were unable to divide, the number of neural precursor cells was seriously 
insufficient, and the differentiation of mature neurons was severely affected, thereby affecting the development of brain tissues [66]. The above studies revealed that methylation and demethylase-mediated posttranscriptional m6A modification play key roles in the development of the mammalian central nervous system, especially cerebellar development.

\section{m6A modification involvement in the development of nerve cells and neural regulation in adults}

After axon injury, the level of m6A modification in nerve cells increases, and the protein translation efficiency of many related genes, including axonal regenerationrelated genes, significantly improves [63]. Studies have shown that the expression of the important "eraser" of m6A, FTO, is highest in the cerebral cortex and is highly expressed in mature neural stem cells and neurons [67] and that FTO deletion seriously damages the neurogenesis and cognitive function of adults [68]. Furthermore, FTO has a highly dynamic expression profile, and researchers suspect that FTO may play a key role in the central nervous system by regulating the expression of m6A-modified nerve-related genes. In addition, RNA methylation also plays a role in the regulation of synaptogenesis in some areas to participate in learning and memory [69-72]. Generally, the dynamic equilibrium of RNA chemical modification is the most important feature in nervous system function. m6A RNA modification also plays an important role in the formation, differentiation, and self-renewal process of mature neurons [67].

\section{Other important functions of $\mathrm{m} 6 \mathrm{~A}$ modification}

Studies have shown that if the m6A modification level of mRNAs of suppressor of cytokine signalling (SOCS) family genes declines, mRNA decay of naïve $\mathrm{T}$ cells can be delayed, and the protein expression levels of SOCS1/3 and cytokine-inducible $\mathrm{SH} 2$-containing protein (CISH) can be increased; however, SOCS1/3 and CISH overexpression directly leads to the inhibition of the downstream IL-7/ STAT5 signalling pathway and the suppression of the proliferation and differentiation of naïve $\mathrm{T}$ cells while maintaining the activity of $\mathrm{T}$ cells [73]. Therefore, m6A RNA modification plays an important role in T-cell homeostasis. In addition, a new study showed that m6A preferentially deposited on the 5' end of nascent transcripts under the action of heat shock proteins. In the UTR, e.g., in HSPH1, m6A modification was increased at the 5'-untranslated region (5'-UTR) to enhance cap-independent translation initiation [74]. In general, the association between 5'-UTR methylation and independent translation reveals the potential relationship between the heat shock response and m6A. We've sorted it out some m6A modification regulators and their major biological functions (Table 1).

\section{Characteristics of $\mathrm{m6A}$ modification in gliomas}

Gliomas are the most common primary malignant tumour in the central nervous system, with characteristics of high malignancy and poor prognosis. Their incidence accounts for $80 \%$ of all brain tumours. Despite the use of a variety of high-intensity treatment regimens, such as surgery combined with chemoradiotherapy, the median survival time of patients with GBM is still only $12-15$ months, and only $3 \%-5 \%$ of patients have a survival time longer than 3 years [1-3]. Therefore, exploring the biological origin and occurrence of gliomas and finding potential diagnostic and therapeutic targets have been the focus of research in the field of molecular biology.

$\mathrm{Li}$ et al. recently reported that $\mathrm{m}^{6} \mathrm{~A}$ methylation is reduced in glioma tissues, and that ectopically increasing $\mathrm{m}^{6} \mathrm{~A}$ levels by METTL3 overexpression in one glioma cell line could impair its proliferation and migratory ability, while increasing apoptosis [77]. But they did not dig into the mechanism through which this epitranscriptomic modification may affect glioblastoma growth.

Cui's group addressed the above point and described the involvement of $\mathrm{m}^{6} \mathrm{~A}$ RNA methylation and of $\mathrm{m}^{6} \mathrm{~A}-$ related proteins in glioblastoma in 2017 [78]. The model they chose were glioblastoma stem cells (GSCs), considered the initiating cells of glioblastoma, usually enriched in restricted niches and deemed responsible not only for glioblastoma onset but also for its resistance to therapy and eventual recurrence [79].

But on the other hand, Liu et al. found that WTAP expression predicts poor prognosis in malignant glioma patients [80]. As WTAP is a crucial interactor of the methyltransferase complex, so this works suggested that $\mathrm{m}^{6} \mathrm{~A}$ modification related enzymes and $\mathrm{m}^{6} \mathrm{~A}$ methylation processes may play an oncogenic role in glioma [81]. Visvanathan et al. published the first mechanistic work linking $\mathrm{m}^{6} \mathrm{~A}$ modification and oncogenesis in glioblastoma. They studied the levels of $\mathrm{m}^{6} \mathrm{~A}$ RNA methylation in three GSC lines and showed that they were reduced uponin vitro differentiation. Moreover, they also found that METTL3 mRNA was clearly more abundant in GSCs compared to counterparts [82, 83].

To sum up, the expression of $\mathrm{m} 6 \mathrm{~A}$ in glioma is different. This indicates that m6A modification may not only promote cancer but also inhibit it during the occurrence and development of glioma. So there's been a lot of interests and researches from biomedical scientists.

\section{Writers and gliomas}

GSCs are a group of cells with the ability to promote tumour growth and invasion and have strong resistance to both radiotherapy and chemotherapy. Therefore, the presence of GSCs indicates a poor prognosis for patients with GBM [84]. One study showed that in GSCs, the 
Table 1 m6A modification regulators and their major biological functions

\begin{tabular}{|c|c|c|c|}
\hline Category & m6A regulator & Main functions & References \\
\hline \multirow[t]{5}{*}{ Writers } & METTL3 & $\begin{array}{l}\text { miRNA regulates mRNA methylation through sequence complementation and cell } \\
\text { reprogramming }\end{array}$ & [47] \\
\hline & METTL3 & Mediates m6A modification involved in the regulation of spermatogenesis in mouse & [59] \\
\hline & METTL3 & Mediates m6A modification involved in the regulation of mouse cerebellar development & [63] \\
\hline & $\begin{array}{l}\text { METTL14, WTAP, VIRMA, } \\
\text { RBM15, ZC3H13, METTL16 }\end{array}$ & m6A methyltransferase complex component identification & [9] \\
\hline & WTAP, METTL3, METTL14 & $\begin{array}{l}\text { WTAP, as a regulatory subunit, regulates the localization and substrate binding capacity } \\
\text { of the catalytic subunits of the METTL3/METTL14 complex }\end{array}$ & [26] \\
\hline \multirow[t]{3}{*}{ Erasers } & FTO & As the first discovered demethylase, FTO can catalyse the demethylation of m6Am and m1A & [75] \\
\hline & FTO & $\begin{array}{l}\text { Mediates m6A modification that can serve as a novel cis element to regulate mRNA splicing } \\
\text { and adipocyte precursor cell differentiation }\end{array}$ & [48] \\
\hline & ALKBH5 & $\begin{array}{l}\text { The second discovered demethylase; participates in the regulation of mRNA nuclear export } \\
\text { and mouse sperm development }\end{array}$ & [33] \\
\hline \multirow[t]{9}{*}{ Readers } & YTHDC1 & YTHDC1 directly interacts with SRSF3 and SRSF10 to regulate alternative mRNA splicing & [35] \\
\hline & YTHDC1 & $\begin{array}{l}\text { YTHDC1 interacts with SRSF3 and RNA nuclear export factor } 1 \text { (NXF1) to regulate mRNA } \\
\text { nuclear export }\end{array}$ & [37] \\
\hline & YTHDF1 & $\begin{array}{l}\text { YTHDF1 directly interacts with the translation initiation complex to promote the translation } \\
\text { efficiency of m6A-modified RNA substrate }\end{array}$ & [34] \\
\hline & YTHDF2 & Mediates m6A modification involved in the regulation of mRNA decay & [44] \\
\hline & YTHDF2, METTL3 & $\begin{array}{l}\text { Mediates m6A modification involved in the regulation of the differentiation of haematopoietic } \\
\text { stem cells }\end{array}$ & [57] \\
\hline & YTHDF1 and YTHDF3 & YTHDF1 synergizes with YTHDF3 to regulate mRNA translation & [76] \\
\hline & YTHDF2 and YTHDF3 & YTHDF2 synergizes with YTHDF3 to mediate mRNA decay & [36] \\
\hline & YTHDC2 & YTHDC2 regulates mRNA translation or decay and mouse spermatogenesis & [38] \\
\hline & IGF2BP1/2/3 & Participates in m6A modification-mediated mRNA stability and translation & [42] \\
\hline
\end{tabular}

expression levels of METTL3 increased, and the expression levels of METTL14 and ALKBH5 decreased, while FTO did not show significant changes. By installing m6A on the SOX2 3'-untranslated region (3'-UTR), METTL3 mediates GSC maintenance and dedifferentiation by regulating the stability of SOX 2 mRNA. The complete structure of METTL3 and human antigen R (HuR) is critical for maintaining this process. In addition, METTL3 knockdown inhibited GSC growth and neurosphere formation and reduced the expression levels of stem cell-specific markers, stage-specific embryonic antigen-1 (SSEA1), and glioma reprogramming factors (including POU class 3 homeobox 2 (POU3F2), oligodendrocyte transcription factor 2 (OLIG2), spalt like transcription factor 2 (SALL2) and SOX2). Of which, SOX2 has a high affinity for METTL3 [82].

The deep sequencing of m6A and mRNA showed that the knockdown of METTL3 and/or METTL14 led to the upregulation of oncogenes and genes coding downstream proteins, including ADAM metallopeptidase domain 19 (ADAM19), EPH receptor A3 (EPHA3), Kruppel-like factor 4 (KLF4) and tumour-inhibiting factors, resulting in the inhibition of GSC growth and selfrenewal [85]. METTL3 overexpression or treatment with the FTO inhibitor MA2, the ethyl ester form of meclofenamic acid (MA), can cause an increase in m6A levels in GBM cells [78]. However, another study reported the opposite effect of METTL3 in GBM; this effect was related to a decrease in m6A levels during differentiation. Silencing METTL3 expression in GBM can significantly inhibit tumour growth and prolong mouse survival time, which is consistent with clinical observations that an increase in METTL3 expression is consistent with the poor survival of patients with GBM. Further studies on the mechanisms of action have shown that METTL3 is involved in the RNA processing and carcinogenic pathways of GSCs and has a variety of complexities. METTL3 plays a major role in m6A modification in GSCs and participates in the expression and alternative splicing of GSC-specific genes. In addition, METTL3 reduced A-to-I RNA editing by downregulating ADAR and ADAMRB1 and increased the editing abundance of C-U RNA by upregulating apolipoprotein $B$ mRNA editing enzyme catalytic subunit 1 (APOBEC1) and APOBEC3A [59].

METTL3 expression is upregulated in GSCs and weakens during differentiation. SOX2 was identified as an important target of METTL3-mediated m6A, whereas METTL3 promoted the recruitment of HuR to m6Amodified SOX2 mRNA and enhanced SOX2 stability 
[85]. In addition, after the downregulation of METTL3 expression, GSCs showed strong radiosensitivity and a weak DNA repair capacity [82]. Therefore, the above studies also revealed that METTL3-mediated m6A modification was important in GSC maintenance and radiotherapy resistance. As a zinc finger protein, zinc finger $\mathrm{CCCH}$-type containing 13 (ZC3H13), is also an important regulator in the m6A-METTL-associated complex (MACOM) and can anchor WTAP, virilizer and Hakai in the nucleus [78]. A recent study showed that the ZC3H13 mutation and retinoblastoma 1 (RB1) mutation could replicate human GBM in a mouse model. In addition, the $\mathrm{ZC} 3 \mathrm{H} 13$ mutation also changed the gene expression profile of the RB1 mutant to enhance the resistance of GBM tumours to TMZ [86].

In addition, WTAP is overexpressed in GBM, and WTAP enhances the proliferation, migration, invasion, and tumourigenicity of GBM cells in xenografts by mediating the phosphorylation of epidermal growth factor receptor (EGFR) and protein kinase $\mathrm{B}$ (AKT). In addition, WTAP also regulates the expression of certain genes associated with cancer cell movement, such as chemokine ligand 2 (CCL2), CCL3, matrix metalloproteinase 3 (MMP3), lysyl oxidase like 1 (LOXL1), hyaluronic acid synthase 1 (HAS1) and thrombospondin 1 (THBS1) [81]. High WTAP expression is an independent prognostic factor that is positively correlated with age and World Health Organization (WHO) classification and indicates poor overall survival in GBM patients [80]. Cell-based experiments have shown that WTAP plays an important role in the miR-29a/Quaking isoform 6 (QKI6) axis-mediated inhibition of cell proliferation, migration, and invasion as well as a downstream target for promoting GSC apoptosis [87].

In addition to the direct impact on pluripotent genes, MeRIP-seq analyses based on m6A-Seq techniques showed that m6A-modification peaks tend to be enriched in metabolic pathway-related transcripts [88]. METTL3 can cause changes through the downregulation of adenosine deaminase 1 (ADAR1) and apolipoprotein B mRNA expression, e.g., a reduction in editing events such as adenosine to inosine (A to I) and cytidine to uridine ( $\mathrm{C}$ to $\mathrm{U}$ ) (such as APOBEC3A) in GSCs [89]. In addition, gene ontology analysis indicated that the direct target of METTL3 seems to be enriched in some major oncogenic pathways, including the Notch signalling pathway, vascular endothelial growth factor (VEGF) signalling pathway, angiogenesis, glycolysis and the Hedgehog signalling pathway; the indirect target is enriched in the RAS pathway, mitogen-activated protein kinase (MAPK) pathway, G-protein coupled receptor (GPCR) pathway, cadherin signalling pathway and cell cycle [89]. In addition, in GSCs, METTL3-mediated m6A modification can also affect expression levels of serine and arginine rich splicing factors (SRSF) by upregulating $\mathrm{BCL}-\mathrm{X}$ or nuclear receptor corepressor 2 and can prevent YTHDC1-dependent nonsense-mediated mRNA decay (NMD) [88]. Compared with protein-coding genes, METTL3-mediated m6A-tagged lncRNAs are also highly expressed in GSCs. Furthermore, the m6A marker in the 3'-UTR appears to block the binding process of microRNA-related genes in GSCs [89].

In summary, m6A writers are critical for the occurrence and development of GBM, and most are upregulated in GBM and show carcinogenic effects by regulating specific signalling pathways, especially helping to maintain cell stemness. However, some opposite findings indicate that the expression of some writers in GBM is downregulated and that some writers may have anticancer properties. Therefore, the existence of this conflict provides more research possibilities on the role of m6A modification-related methylation in the biological pathogenesis of gliomas.

\section{Erasers and gliomas}

Similar to writers, m6A erasers also play vital roles in GBM. The latest research shows that ALKBH5 is elevated in GSCs, enhancing cell self-renewal, proliferation and tumourigenicity [78]. In terms of a mechanism, ALKBH5 demethylates m6A-modified bases and enhances the expression level of the key target gene forkhead box protein M1 (FOXM1) in GBM patients by reducing the abundance of $\mathrm{m} 6 \mathrm{~A}$ in the target mRNA transcript (especially in the 3'-UTR) [90].

As an important functional target of ALKBH5, FOXM1 overexpression can reverse the function of ALKBH5 or inhibit FOXM1 long noncoding RNA antisense (FOXM1-AS) and restore GSC tumour growth. FOXM1-AS is a IncRNA on human chromosome 12 that is opposite to and partially overlaps with FOXM1. FOXM1-AS can promote the interaction between ALKBH5 and FOXM1 nascent transcripts, thereby promoting the recruitment of HuR. In general, under the combined action of FOXM1-AS, ALKBH5 enhances the self-renewal and proliferation of GSCs by regulating the expression of FOXM1 and promoting the occurrence and development of GBM [90]. On the other hand, ALKBH5 knockdown inhibits the proliferation of GSCs, while wild-type ALKBH5 rescues the proliferation of GSCs. After ALKBH5 knockdown, the m6A level in nascent FOXM1 transcripts is elevated, and the binding of FOXM1 pre-mRNA to HuR is reduced; therefore, the recruitment of HuR to m6A-modified RNA is crucial for stabilizing FOXM1 mRNA [90].

Su's study showed that the inhibition of FTO hindered the self-renewal ability and carcinogenicity of GBM stem cells in vitro and in mouse models. FTO plays carcinogenic roles through maintaining the stability of gliomas, 
especially the stability of oncogene homologues (c-Myc) and CCAAT-enhancer-binding protein- $\alpha$ (CEBPA) transcripts in IDH1/2 mutant gliomas. In addition, the inhibitory effect of MA2 on FTO significantly increases the tumourigenicity of GSC-transplanted mice [75, 78]. The above evidence also potentially reveals that FTO may be a promising target for the drug treatment of GBM.

\section{Readers and gliomas \\ YTHDF and YTHDC family}

The YTHDF and YTHDC series are the most important code readers for m6A modification, as they include a YTH domain that can bind to RNA. They exhibit different functions: YTHDC1 mediates mRNA splicing; YTHDF2, YTHDF3 and YTHDC2 mediate mRNA decay; YTHDF1, YTHDF3 and YTHDC2 mediate mRNA translation; and YTHDC2 and YTHDC2 mediate RNA structure [91]. Recently, a study by Li et al. showed that these important m6A-related proteins are involved in the development of GBM. After YTHDC1 knockdown, proliferation was significantly reduced in U87 cells with METTL3 overexpression but not in control cells. In addition, for the W377A/W428A mutant with METTL3 overexpression, YTHDC1 failed to promote the ability of U87 cells to form spheres, indicating that YTHDC1 relies on its $\mathrm{m} 6 \mathrm{~A}$ binding activity to promote the functional phenotype of GBM [88].

\section{IGF2BP family}

Another m6A reader protein family, the IGF2BP family (IGF2BP1/2/3), inhibits the decay of m6A-modified transcripts and promotes their translation [42]. For a long time, these proteins have been considered important regulatory factors in the pathogenesis of GBM, even though some functions are not directly related to carcinogenesis. For example, IGF2BP1 can promote the proliferation and invasion of GBM cells by stabilizing the mRNA transcripts of its target genes, including cMyc, Ki-67, phosphatases and tensin homologue (PTEN) and CD44 (cell-surface glycoprotein 44) [92, 93].

Studies have shown that IGF2BP2 is upregulated in GBM tissues, promoting the proliferation, migration, invasion, and epithelial-mesenchymal transition (EMT) of GBM cells through the regulation of insulin-like growth factor 2 (IGF2) activity while further activating the phosphoinositide 3-kinases (PI3K)/AKT signalling pathway. In addition, IGF2BP2 inhibition can cause an increase in the sensitivity of GBM to TMZ [94]. Another study showed that IGF2BP2 binds to the miRNA recognition elements (MREs) of lethal-7 (let-7) and blocks let-7 target gene silencing, which is LIN28-independent, including high mobility group AT-hook 1 (HMGA1), HMGA2, cyclin D2 (CCND2) and ribonucleotide reductase regulatory subunit M2 (RRM2), thus maintaining the stemness of GSCs [95]. During this process, IGF2BP2 is responsible for miRNA maturation, and it also interacts with lncRNAs such as hypoxia inducible factor 1 alphaantisense RNA 2 (HIF1A-AS2), which can specifically induce hypoxia to maintain the expression level of its target gene HMGA1, eventually promoting GSC proliferation, self-renewal and the reprogramming of hypoxiadependent molecules [96]. In addition, IGF2BP2 also interacts with mRNAs and proteins. For example, IGF2BP2 can bind to several mRNAs, including mitochondrial cytochrome $\mathrm{C}$ oxidase subunit 7B (COX7B), NADH dehydrogenase iron-sulfur protein 7 (NDUS7), and NADH dehydrogenase, to promote oxidative phosphorylation in GSCs [97].

The mRNA and protein expression levels of IGF2BP3 are upregulated in GBM but not significantly upregulated in low-grade astrocytomas [98]. According to the gene expression microarray analysis of 9 pilomyxoid astrocytoma (PMA) and 13 pilocytic astrocytoma (PA) from lower and upper loci, the expression level of IGF2BP3 in malignant astrocytoma is significantly increased [99]. In addition, gene chip analysis in glioma cells indicated that IGF2BP3 mediates the association between direct targets at the transcriptome level and processes related to the cell cycle as well as the association between direct targets at the translatome level and apoptosis-related pathways [100]. IGF2BP3 also induced EMT by downregulating the expression of E-cadherin and upregulating the expression levels of $\mathrm{N}$-cadherin, vimentin and MMP-9, thereby promoting cell proliferation, migration and invasion [101]. In addition, IGF2BP3 activates the PI3K/MAPK pathway by binding to the 5'UTR of IGF-2 mRNA to activate its translation, thereby promoting cell proliferation, anchorage-independent growth, invasion, and chemoresistance [98]. IGF2BP3 also stimulates the migration of glioma cells by enhancing the translation of p65 (RELA), which is a subunit of the nuclear factor-kappa B (NF- $\mathrm{kB})$ heterodimer, and p65 can also transcriptionally activate IGF2BP3 to form a feedback loop [102].

\section{hnRNPA2B1 and hnRNPC family}

A study of SOX2 protein interactions showed that hnRNPA2B1 and hnRNPC could interact with SOX2 in GBM, suggesting that they might play a key role in maintaining the stemness of GSCs [103]. Heterogeneous nuclear ribonucleoproteins A2/B1 (hnRNPA2B1) is an important regulator of mRNA metabolism and transport in cells. Its downstream protein is highly expressed in glioma tissues and is associated with the histologic glioma grade and a poor prognosis [104]. hnRNPA2B1 may promote the proliferation, migration, and invasion of GBM cells through the downregulation of tumour 
suppressor factors, enhance chemoresistance to TMZ, and protect cells from apoptosis and damage caused by reactive oxygen species (ROS) [105].

In addition, hnRNPC is an important physiological modulator for 3'-UTR processing and miRNA maturation as well as a modulator for neoplastic disease [106]. hnRNPC has higher expression levels in higher-grade GBM; hnRNPC directly binds to miR-21 (mainly primiR-21) and promotes miR-21 processing against programmed cell death 4 (PDCD4), which is an important regulator of cell apoptosis and survival. PDCD4 subsequently promotes the activation of AKT and p70 S6 kinase (p70S6K) and then enhances the migration and invasion activity of tumour cells, increases cell proliferation, and protects GBM cells from apoptosis [107]. These results indicate that hnRNPA2B1 and hnRNPC may be important m6A "readers" that are closely related to the biogenesis of GBM. Finally, we mapped the mechanism of action of m6A-modified important proteins associated with the biological behavior of glioma cells (Fig. 3).

\section{Clinical significance of m6A modification in gliomas \\ m6A modification and disease diagnosis}

Epigenetic alterations are considered promising markers for GBM diagnosis. In addition, some epigenetic statuses indeed explain the outcome of GBM [108-111]. For example, the epigenetic silencing of O-6-methylguanineDNA methyltransferase (MGMT) significantly affected the TMZ treatment effect in GBM patients. Therefore, the methylation status of the MGMT promoter is an important biological marker used to predict GBM patient survival and GBM response to TMZ $[112,113]$.

As an important chemical modification to RNA, regular changes in $\mathrm{m} 6 \mathrm{~A}$ can also predict the prognosis of patients with GBM or be used for the diagnosis of GBM. Related studies have shown that m6A in RNA from peripheral blood is a biomarker of gastric cancer [114]. The methylation level of miRNAs is also a potential diagnostic biomarker for early gastrointestinal cancer [115]. ALKBH5 and FTO, which act as m6A erasers, have also been proven to be prognostic biomarkers for patients

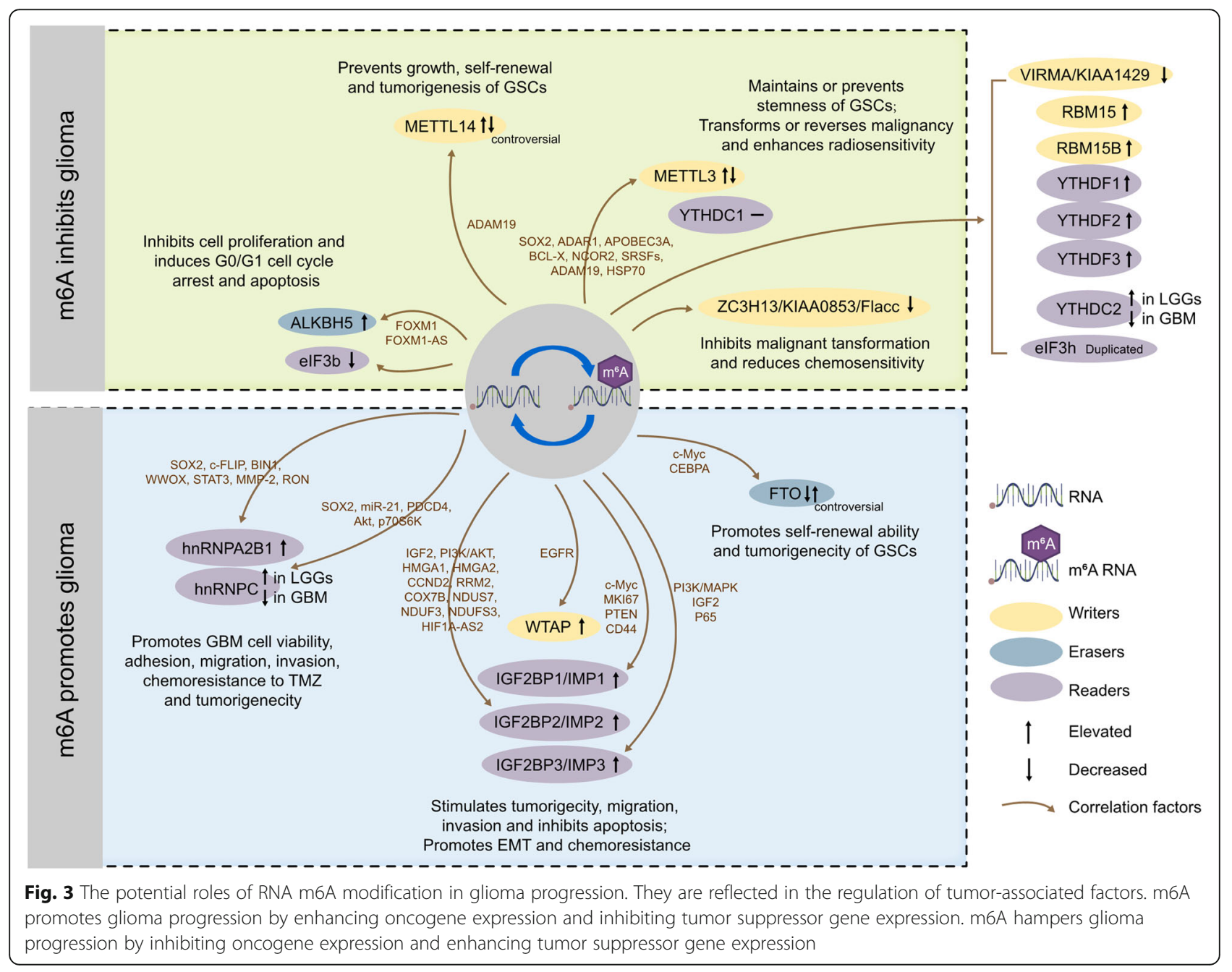


with renal clear cell carcinoma [116]. Therefore, for central nervous system tumours, the identification of modifications to RNA from peripheral blood or cerebrospinal fluid may be a promising method for GBM diagnosis.

\section{Expression of the $\mathrm{m} 6 \mathrm{~A}$ modifier is related to the clinicopathological features of gliomas}

Considering the important biological functions of m6A modification-related proteins involved in the occurrence and development of tumours, some researchers conducted genomic profiling-based data mining and bioinformatics analysis to systematically investigate the relationship between each type of m6A modulator and pathological features of gliomas, including WHO classification, isocitrate dehydrogenase (IDH) classification and $1 \mathrm{p} / 19 \mathrm{q}$ status. The results showed that the expression level of most m6A modulators was significantly correlated with the WHO histologic grade and corresponding classification. Through quantitative analysis, the expression levels of WTAP, YTHDF, ALKBH5 and FTO were significantly correlated with the histologic grade, the expression abundances of WTAP, YTHDF and ALKBH5 were positively correlated with the histologic grade, and the expression abundance of FTO was negatively correlated with the histologic grade [117].

\section{Potential therapeutic significance of $\mathrm{m} 6 \mathrm{~A}$ modification in gliomas}

The covalent modification of DNA, histones and other proteins has shown potential as a cancer treatment. Some epigenetic drugs, such as suberanilohydroxamic acid (SAHA), romidepsin, Belinostat and Panobinostat, have been approved by the U.S. Food and Drug Administration (FDA). Moreover, chidamide has also been approved by the Chinese National Medical Products Administration for the treatment of certain T-cell lymphomas or multiple myeloma. In addition, many other clinical trials of epigenetic drugs are also underway [118]. Drugs targeting protein posttranslational modifiers (such as E3 ubiquitin-protein ligase), including Sphase kinase associated protein 2 (SKP2), speckle type $\mathrm{BTB} / \mathrm{POZ}$ protein (SPOP), cellular inhibitor of apoptosis 2 (cIAP) and anaphase-promoting complex/cyclosome $(\mathrm{APC} / \mathrm{C})$, have also been evaluated in clinical practice and preclinical applications [119].

$\mathrm{m} 6 \mathrm{~A}$ is the most common covalent modification of RNA at the posttranscriptional level. Scientists are exploring its therapeutic potential in malignant tumours such as GBM. Currently, some pharmaceutical companies are planning to develop a METTL3-METTL14 complex and a small molecule inhibitors of ADAR [120]. Several compounds with piperidine or piperazine rings have very high cooperative binding efficiency with METTL3-METTL14WTAP complexes, thereby activating RNA methylation
[121]. Recently, METTL3 small molecule inhibitors have also shown the ability to inhibit the progression of acute myeloid leukaemia (AML) in the body [122]. Based on the abovementioned latest research, it is believed that m6A modification-related targeted therapies for gliomas will be developed.

\section{m6A modification and prognosis of patients with gliomas}

Some researchers conducted a univariate Cox regression analysis on the expression levels of m6A-related proteins in the Chinese Glioma Genome Atlas (CGGA) database and found that high-risk genes mainly include ALKBH5, YTHDF1, YTHDF2, HNRNPC, RNA binding motif protein 15 (RBM15), KIAA1429 and WTAP and that the protective genes are mainly FTO, YTHDC1, ZC3H13 and METTL3. Among the genes with predictive value in adult low-grade gliomas (LGGs), the expression levels of YTHDF2, WTAP, ALKBH5, RBM15, KIAA1429, HNRN PC, YTHDF1 and FTO were significantly correlated with the overall survival (OS) of IDH-mutated glioma patients. In summary, YTHDF2, KIAA1429, HNRNPC, and YTHDF1 had predictive value in LGB with wildtype IDH, and FTO, YTHDF2, and RBM15 had predictive value in GBM with wild-type IDH; FTO also had predictive value in GBM with mutated IDH [117].

In addition, another univariate and multivariate Cox regression analysis showed that risk score, $1 \mathrm{p} / 19 \mathrm{q}$ sequence, IDH status, age and WHO classification were all associated with OS. After including these factors in the multivariate Cox regression, risk scores and WHO classification were still significantly correlated with OS $(\mathrm{P}<$ 0.001). Further multiple regression analysis showed that risk score $(\mathrm{P}=0.027)$, IDH status $(\mathrm{P}<0.001)$, age $(\mathrm{P}<$ $0.001)$ and $\mathrm{WHO}$ classification $(\mathrm{P}=0.005)$ were still significantly correlated with OS. In summary, the risk score derived from the RNA m6A modulator can independently predict the prognosis of glioma patients. In addition, for WHO grades II and III gliomas, the OS of patients with high risk scores was significantly shorter than that of patients with lower risk scores. Through comprehensive analysis of the WHO classification, risk score also had predictive value for gliomas. In addition, according to the CGGA and The Cancer Genome Atlas (TCGA) databases, GBM patients with high risk scores were also more sensitive to TMZ treatment and had relatively lower drug resistance [117].

\section{Discussion and outlook}

There is a correlation between the degree of m6A RNA methylation and the differentiation of GSCs and between the effect of METTL3 knockdown on in vitro selfrenewal and in vivo tumourigenicity of glioblastoma in mice. GSCs and their derived tumours can be divided into subtypes based on different characteristics and 
different aggressiveness and responsiveness to therapy. Research on m6A modification and oncology is still an emerging field. Therefore, all cited papers are based on a very limited number of cell models, and the results may be affected by variability. One study analysed 7 GSC lines using RNA-seq and compared the normal human brain with an established GBM cell line; the study did not find any clear evidence (e.g., author, reader, eraser, etc.) to prove the differential expression of mRNA coding proteins involved in m6A methylation. However, the controversial results were not discredited, and some genes or proteins involved in mRNA expression were still considered potential prognostic markers of GBM.

However, for this mechanism, it seems more necessary to clarify which mRNAs are hypermethylated or hypomethylated and which readers identify methylation under certain circumstances. It has been demonstrated that IGF2BPs are carcinogenic in glioblastoma, especially in GSCs, in which their function as specific target mRNA stability enhancers is related to their ability to induce or maintain glioblastoma growth. By correlating these data with currently known m6A readers, we can gain a deeper understanding of other molecular mechanisms by which these proteins may be involved in glioblastoma tumourigenesis.

Wu et al. recently published a study demonstrating that the m6A reader PRRC2a plays a key role in the formation of oligodendrocytes and myelination by binding to methylated adenosine in glioblastoma cells. This binding can stabilize OLIG2 mRNA, thereby stabilizing Olig2 protein production. In contrast, FTO demethylates OLIG2 mRNA at this site, thereby promoting its decay and Olig2 protein consumption. This study focused on oligodendrocytes in physiological and pathologically low-myelination conditions, but it should be noted that in addition to playing a major role in the development of oligodendrocytes, Olig2 protein also plays a role in GBM cell reprogramming and the genotoxicity and phenotypic plasticity of tumours. In addition, Olig2 expression is a recognized marker for the proneural subtype of GBM. More importantly, Olig2 protein is synergistic with Sox2, Pou3f2 and Sall2 and is a key transcription factor of glioma initiating cells [123]. This observation is only an example that partially elucidates how the proteins involved in transcriptome regulation are transferred under specific pathological conditions, especially in GBM. This field is worthy of further in-depth studies. Based on the information regarding transcriptome modifications in gliomas obtained from recent studies, we may have only revealed the tip of the iceberg, and in the future, we will definitely find more complex mechanisms of precise regulation of the transcriptional fate of glioma cells.

\section{Conclusions}

In summary, although the correlation between m6A modification and oncology, as a hotspot in the field of biomedicine, has been extensively explored, most studies concentrated on gene sequencing analysis, differential expression analysis, and modification site analysis. There are few studies on the functional phenotypes and mechanisms of action at the cell level, but studies in this field are likely to be key to revealing the origin of tumours, especially the origin and development of malignant tumours. With the rapid development of molecular biology technology, especially single-cell sequencing and thirdgeneration sequencing, the answer of how RNA methylation modification affects the biological behaviour of gliomas will gradually become clear. It provides new insights for the early diagnosis of, histologic grading of, and targeted therapy for gliomas. It also points to a new direction for the study of other neoplastic diseases. As a star molecule in RNA post-transcription modification research, exploring m6A modification might decipher the molecular mechanism of many diseases, serving as a new medical breakthrough anticipated by biomedical scientists.

\section{Abbreviations}

GBM: Glioblastoma; TMZ: Temozolomide; m6A: N6-methyladenine; mRNA: Messenger RNA; m6A-seq: M6A RNA immunoprecipitation sequencing; HPLC-MS/MS: High-performance liquid chromatography-tandem mass spectrometry; MeRIP-seq: Methylated RNA immunoprecipitation followed by high-throughput sequencing; PA-m6A-Seq: Photo-crosslinkingassisted m6A-sequencing; SCARLET: Site-specific cleavage and radioactivelabelling followed by ligation-assisted extraction and thin-layer chromatography; miCLIP: M6A individual nucleotide resolution crosslinking immunoprecipitation; miRNA: Micro RNA; IncRNA: Long noncoding RNA; circRNA: Circular RNA; rRNA: Ribosomal RNA; tRNA: Transfer RNA; snoRNA: Small nucleolar RNA; METTL3: Methyltransferase like 3; WTAP: Wilms' tumour 1-associating protein; SAM: S-adenosylmethionine; FTO: Fat mass and obesity-associated gene; ALKBH5: AlkB homologue 5; YTHDF1: YTH N6methyladenosine binding protein 1; YTHDC1: YTH domain containing 1; SRSF3: Serine-rich splicing factor 3; IGF2BP: Insulin-like growth factor-II mRNA binding protein ; P body: Processing body; EHT: Endothelial-to-

haematopoietic transition; Notch1: Notch homologue protein 1; HSPC: Haematopoietic stem cell; ESC: Embryonic stem cell; ZFP217: Zinc finger protein 217; SOX2: Sex determining region Y box 2; KLF4: Kruppel-like factor 4; HE: Haematoxylin-eosin; IL-7: Interleukin-7; STAT5: Signal transducer and activator of transcription 5; CGC: Cerebellar granule cell; EGL: External granular layer; SOCS: Suppressor of cytokine signalling; CISH: Cytokineinducible SH2-containing protein; 5'-UTR: 5'-untranslated region; GSC: Glioblastoma stem cell; 3'-UTR: 3'-untranslated region; HuR: Human antigen R; SSEA1: Stage-specific embryonic antigen 1; POU3F2: POU class 3 homeobox 2 ; OLIG2: Oligodendrocyte transcription factor 2; SALL2: Spalt like transcription factor 2; ADAM19: ADAM metallopeptidase domain 19;

EPHA3: EPH receptor A3; MA: Meclofenamic acid; APOBEC1: Apolipoprotein B mRNA editing enzyme catalytic subunit 1; ZC3H13: Zinc finger CCCH-type containing 13; MACOM: M6A-METTL-associated complex;

RB1: Retinoblastoma 1; EGFR: Epidermal growth factor receptor;

CCL2: Chemokine ligand 2; MMP3: Matrix metalloproteinase 3; LOXL1: Lysyl oxidase like 1; HAS1: Hyaluronic acid synthase 1; THBS1: Thrombospondin 1 ; WHO: World Health Organization; QKI-6: Quaking isoform-6;

ADAR1: Adenosine deaminase 1; VEGF: Vascular endothelial growth factor; MAPK: Mitogen-activated protein kinase; GPCR: G-protein coupled receptor SRSF: Serine and arginine rich splicing factor; NMD: Nonsense-mediated mRNA decay; FOXM1: Forkhead box protein M 1; CEBPA: CCAAT-enhancerbinding protein-a; PETN: Phosphatases and tensin homologue; CD44: Cellsurface glycoprotein 44; EMT: Epithelial-mesenchymal transition; IGF2: Insulinlike growth factor 2; PI3K: Phosphoinositide 3-kinases; MRE: MiRNA recognition element ; let-7: Lethal-7; HMGA1: High mobility group AT-hook 1; CCND2: Cyclin D 2; RRM2: Ribonucleotide reductase regulatory subunit M 2; 
HIF1A-AS2: Factor 1 alpha-antisense RNA 2; COX7B: Cytochrome C oxidase subunit 7 B; NDUS7: NADH dehydrogenase iron-sulfur protein 7;

PMA: Pilomyxoid astrocytoma; PA: Pilocytic astrocytoma; NF-KB: Nuclear factor-kappa B; hnRNPA2B1: Heterogeneous nuclear ribonucleoproteins A2/ B1; ROS: Reactive oxygen species; PDCD4: Programmed cell death 4; p70S6K: P70 S6 kinase; MGMT: Methylguanine-DNA methyltransferase; IDH: Isocitrate dehydrogenase; SAHA: Suberanilohydroxamic acid ; FDA: Food and drug administration; SKP2: S-phase kinase associated protein 2; SPOP: Speckle type BTB/POZ protein; CIAP: Cellular inhibitor of apoptosis; APC/C: Anaphase-promoting complex/cyclosome; AML: Acute myeloid leukaemia; CGGA: Chinese Glioma Genome Atlas; RBM15: RNA binding motif protein 15; LGG: Adult low-grade glioma; OS: Overall survival; TCGA: The Cancer Genome Atlas

\section{Acknowledgements}

We would like to give special thanks to Ruijuan Ren, the curator of Hebei University library, for her guidance and assistance in the literature retrieval process of this paper.

\section{Authors' contributions}

$\mathrm{YHZ}$ and XCG were the major contributors to the writing and revision of the manuscript. QL, JLX and YLT collected the related references and participated in discussions. MLX and JS revised this article critically for important intellectual content. FLL, CF and HW gave approval for the final version of the manuscript All authors have read and approved the final manuscript.

\section{Funding}

The present study was financially supported by grants from the Natural Science Foundation of Hebei Province in 2020 (no.H2020201050); High-level Talents Scientific Research Launched Project of Hebei University(no.521000981301); Government-funded Clinical Medicine Outstanding Talent Training Project(Team) in 2019(no.360017); Science and Technology Capacity Improvement Projects of Hebei University of Chinese Medicine in 2019 (no.KTZ2019019); Outstanding Student Scientific Research Ability Improvement Projects of Hebei University of Chinese Medicine in 2019 (no.YXZ2019002); Graduate Innovative Ability Training Projects of Hebei University of Chinese Medicine in 2020 (no.XCXZZBS2020002); Graduate Innovative Ability Training Projects of Hebei University in 2020 (no.hbu2020bs003); Foundation Project of Affiliated Hospital of Hebei University in 2019 (no.2019Q009).

\section{Availability of data and materials}

The datasets used and analysed during the present study are available from the corresponding author on reasonable request.

\section{Ethics approval and consent to participate}

Not applicable.

\section{Consent for publication}

Not applicable.

\section{Competing interests}

The authors declare that they have no competing interests

\section{Author details}

${ }^{1}$ Department of Neurosurgery, Affiliated Hospital of Hebei University, 071000 Baoding, China. ${ }^{2}$ Faculty of Integrated Traditional Chinese and Western Medicine, Hebei University of Chinese Medicine, 050091 Shijiazhuang, China. ${ }^{3}$ Faculty of Acupuncture-Moxibustion and Tuina, Hebei University of Chinese Medicine, 050200 Shijiazhuang, China. ${ }^{4}$ Department of Pathology, Affiliated Hospital of Hebei University, 071000 Baoding, China. ${ }^{5}$ School of Basic Medicine, Hebei University, 071000 Baoding, China. ${ }^{6}$ Office of Academic Research, Affiliated Hospital of Hebei University, 071000 Baoding, China. ${ }^{7}$ Hebei Key Laboratory of Chinese Medicine Research on Cardio-Cerebrovascular Disease, Hebei University of Chinese Medicine, 050091 Shijiazhuang, China.
Received: 29 July 2020 Accepted: 10 September 2020 Published online: 17 September 2020

\section{References}

1. Louis DN, Perry A, Reifenberger G, von Deimling A, Figarella-Branger D, Cavenee WK, et al. The 2016 World Health Organization Classification of Tumors of the Central Nervous System: a summary. Acta Neuropathol. 2016; 131(6):803-20.

2. Lapointe S, Perry A, Butowski NA. Primary brain tumours in adults. Lancet. 2018;392(10145):432-46.

3. Kristensen BW, Priesterbach-Ackley LP, Petersen JK, Wesseling P. Molecular pathology of tumors of the central nervous system. Ann Oncol. 2019;30(8): 1265-78.

4. Lim M, Xia Y, Bettegowda C, Weller M. Current state of immunotherapy for glioblastoma. Nat Rev Clin Oncol. 2018;15(7):422-42.

5. Touat M, Li YY, Boynton AN, Spurr LF, lorgulescu JB, Bohrson CL, et al. Mechanisms and therapeutic implications of hypermutation in gliomas. Nature. 2020;580(7804):517-23.

6. Chen $X Y$, Zhang J, Zhu JS. The role of $m(6) A$ RNA methylation in human cancer. Mol Cancer. 2019;18(1):103

7. Deng X, Su R, Weng H, Huang H, Li Z, Chen J. RNA N(6)-methyladenosine modification in cancers: current status and perspectives. Cell Res. 2018;28(5): 507-17

8. Dawson MA, Kouzarides T. Cancer epigenetics: from mechanism to therapy. Cell. 2012;150(1):12-27.

9. Wimalasena VK, Wang T, Sigua LH, Durbin AD, Qi J. Using Chemical Epigenetics to Target Cancer. Mol Cell. 2020;78(6):1086-95.

10. Saletore $Y$, Meyer K, Korlach J, Vilfan ID, Jaffrey S, Mason CE. The birth of the Epitranscriptome: deciphering the function of RNA modifications. Genome Biol. 2012;13(10):175.

11. Wang T, Kong S, Tao M, Ju S. The potential role of RNA N6methyladenosine in Cancer progression. Mol Cancer. 2020;19(1):88.

12. Frye M, Jaffrey SR, Pan T, Rechavi G, Suzuki T. RNA modifications: what have we learned and where are we headed? Nat Rev Genet. 2016;17(6):365-72.

13. Shi H, Wei J, He C. Where, When, and How: Context-Dependent Functions of RNA Methylation Writers, Readers, and Erasers. Mol Cell. 2019;74(4):640-50.

14. Dominissini D, Moshitch-Moshkovitz S, Schwartz S, Salmon-Divon M, Ungar $\mathrm{L}$, Osenberg $\mathrm{S}$, et al. Topology of the human and mouse m6A RNA methylomes revealed by m6A-sEq. Nature. 2012;485(7397):201-6.

15. Jia G, Fu Y, Zhao X, Dai Q, Zheng G, Yang Y, et al. N6-methyladenosine in nuclear RNA is a major substrate of the obesity-associated FTO. Nat Chem Biol. 2011;7(12):885-7.

16. Bodi Z, Fray RG. Detection and Quantification of N (6)-Methyladenosine in Messenger RNA by TLC. Methods Mol Biol (Clifton NJ). 2017;1562:79-87.

17. Dominissini D, Moshitch-Moshkovitz S, Schwartz S, Salmon-Divon M, Ungar L, Osenberg S, et al. Topology of the human and mouse m6A RNA methylomes revealed by m6A-sEq. Nature. 2012;485(7397):201-6.

18. Meyer KD, Saletore $Y$, Zumbo P, Elemento O, Mason CE, Jaffrey SR. Comprehensive analysis of mRNA methylation reveals enrichment in $3^{\prime}$ UTRs and near stop codons. Cell. 2012;149(7):1635-46.

19. Chen K, Lu Z, Wang X, Fu Y, Luo GZ, Liu N, et al. High-resolution N(6) -methyladenosine (m(6) A) map using photo-crosslinking-assisted m(6) A sequencing. Angewandte Chemie (International ed in English). 2015;54(5): 1587-90.

20. Liu N, Pan T. Probing RNA Modification Status at Single-Nucleotide Resolution in Total RNA. Methods Enzymol. 2015:560:149-59.

21. Linder B, Grozhik AV, Olarerin-George AO, Meydan C, Mason CE, Jaffrey SR. Single-nucleotide-resolution mapping of $\mathrm{m} 6 \mathrm{~A}$ and $\mathrm{m} 6 \mathrm{Am}$ throughout the transcriptome. Nat Methods. 2015;12(8):767-72.

22. Gilbert WV, Bell TA, Schaening C. Messenger RNA modifications: Form, distribution, and function. Science (New York, NY). 2016;352(6292): 1408-12.

23. Sun $T$, Wu R, Ming $L$. The role of m6A RNA methylation in cancer. Biomed Pharmacother. 2019;112:108613.

24. Coker H, Wei G, Brockdorff N. m6A modification of non-coding RNA and the control of mammalian gene expression. Biochim Biophys Acta Gene Regul Mech. 2019;1862(3):310-8.

25. Ma S, Chen C, Ji X, Liu J, Zhou Q, Wang G, et al. The interplay between m6A RNA methylation and noncoding RNA in cancer. J Hematol Oncol. 2019; 12(1):121. 
26. Ping $X L$, Sun BF, Wang L, Xiao W, Yang X, Wang WJ, et al. Mammalian WTAP is a regulatory subunit of the RNA N6-methyladenosine methyltransferase. Cell Res. 2014;24(2):177-89.

27. Liu J, Yue Y, Han D, Wang X, Fu Y, Zhang L. et al. A METTL3-METTL14 complex mediates mammalian nuclear RNA N6-adenosine methylation. Nat Chem Biol. 2014;10(2):93-5.

28. Liu ZX, Li LM, Sun HL, Liu SM. Link Between m6A Modification and Cancers. Front Bioeng Biotechnol. 2018;6:89.

29. Śledź P, Jinek M. Structural insights into the molecular mechanism of the m(6)A writer complex. Elife. 2016;5:e18434

30. Wang P, Doxtader KA, Nam Y. Structural Basis for Cooperative Function of Mettl3 and Mettl14 Methyltransferases. Mol Cell. 2016;63(2):306-17.

31. Jia G, Fu Y, Zhao X, Dai Q, Zheng G, Yang Y, et al. N6-methyladenosine in nuclear RNA is a major substrate of the obesity-associated FTO. Nat Chem Biol. 2011:7(12):885-7.

32. Mauer J, Luo X, Blanjoie A, Jiao X, Grozhik AV, Patil DP, et al. Reversible methylation of $m(6) A(m)$ in the $5^{\prime}$ cap controls mRNA stability. Nature. 2017; 541(7637):371-5.

33. Zheng G, Dahl JA, Niu Y, Fedorcsak P, Huang CM, Li CJ, et al. ALKBH5 is a mammalian RNA demethylase that impacts RNA metabolism and mouse fertility. Mol Cell. 2013;49(1):18-29.

34. Wang X, Zhao BS, Roundtree IA, Lu Z, Han D, Ma H, et al. N(6)methyladenosine Modulates Messenger RNA Translation Efficiency. Cell. 2015;161(6):1388-99.

35. Xiao W, Adhikari S, Dahal U, Chen YS, Hao YJ, Sun BF, et al. Nuclear m(6)A Reader YTHDC1 Regulates mRNA Splicing. Mol Cell. 2016;61(4): 507-19.

36. Shi H, Wang X, Lu Z, Zhao BS, Ma H, Hsu PJ, et al. YTHDF3 facilitates translation and decay of N(6)-methyladenosine-modified RNA. Cell Res. 2017:27(3):315-28.

37. Roundtree IA, Luo GZ, Zhang Z, Wang X, Zhou T, Cui Y, et al. YTHDC1 mediates nuclear export of N(6)-methyladenosine methylated mRNAs. ELife. 2017;6:e31311

38. Hsu PJ, Zhu Y, Ma H, Guo Y, Shi X, Liu Y, et al. Ythdc2 is an N(6)methyladenosine binding protein that regulates mammalian spermatogenesis. Cell Res. 2017:27(9):1115-27.

39. Sun $T$, Wu R, Ming L. The role of m6A RNA methylation in cancer. Biomed Pharmacother. 2019;112:108613.

40. Ke S, Alemu EA, Mertens C, Gantman EC, Fak JJ, Mele A. et al. A majority of m6A residues are in the last exons, allowing the potential for $3^{\prime}$ UTR regulation. Genes Dev. 2015;29(19):2037-53.

41. Molinie B, Wang J, Lim KS, Hillebrand R, Lu ZX, Van Wittenberghe N, et al. $m(6) A-L A I C-s e q$ reveals the census and complexity of the $m(6) A$ epitranscriptome. Nat Methods. 2016;13(8):692-8.

42. Huang $H$, Weng $H$, Sun W, Qin $X$, Shi $H$, Wu H, et al. Recognition of RNA N(6)-methyladenosine by IGF2BP proteins enhances mRNA stability and translation. Nat Cell Biol. 2018;20(3):285-95.

43. Zhou J, Wan J, Gao X, Zhang X, Jaffrey SR, Qian SB. Dynamic m(6)A mRNA methylation directs translational control of heat shock response. Nature. 2015;526(7574):591-4.

44. Wang X, Lu Z, Gomez A, Hon GC, Yue Y, Han D, et al. N6-methyladenosinedependent regulation of messenger RNA stability. Nature. 2014;505(7481): 117-20.

45. Liu N, Dai Q, Zheng G, He C, Parisien M, Pan T. N(6)-methyladenosinedependent RNA structural switches regulate RNA-protein interactions. Nature. 2015;518(7540):560-4.

46. Liu N, Zhou Kl, Parisien M, Dai Q, Diatchenko L, Pan T. N6-methyladenosine alters RNA structure to regulate binding of a low-complexity protein. Nucleic Acids Res. 2017;45(10):6051-63.

47. Chen T, Hao YJ, Zhang Y, Li MM, Wang M, Han W, et al. m(6)A RNA methylation is regulated by microRNAs and promotes reprogramming to pluripotency. Cell Stem Cell. 2015;16(3):289-301.

48. Zhao $X$, Yang $Y$, Sun BF, Shi $Y$, Yang $X$, Xiao W, et al. FTO-dependent demethylation of N6-methyladenosine regulates mRNA splicing and is required for adipogenesis. Cell Res. 2014;24(12):1403-19.

49. Fustin JM, Doi M, Yamaguchi $Y$, Hida H, Nishimura S, Yoshida M, et al. RNAmethylation-dependent RNA processing controls the speed of the circadian clock. Cell. 2013;155(4):793-806.

50. Xiang Y, Laurent B, Hsu CH, Nachtergaele S, Lu Z, Sheng W, et al. RNA $\mathrm{m}(6)$ A methylation regulates the ultraviolet-induced DNA damage response. Nature. 2017;543(7646):573-6.
51. Aguilo F, Zhang F, Sancho A, Fidalgo M, Di Cecilia S, Vashisht A, et al. Coordination of m(6)A mRNA Methylation and Gene Transcription by ZFP217 Regulates Pluripotency and Reprogramming. Cell Stem Cell. 2015; 17(6):689-704.

52. Geula S, Moshitch-Moshkovitz S, Dominissini D, Mansour AA, Kol N, SalmonDivon $\mathrm{M}$, et al. Stem cells. m6A mRNA methylation facilitates resolution of naïve pluripotency toward differentiation. Science. 2015;347(6225):1002-6.

53. Zhao BS, Wang X, Beadell AV, Lu Z, Shi H, Kuuspalu A, et al. m(6)Adependent maternal mRNA clearance facilitates zebrafish maternal-tozygotic transition. Nature. 2017;542(7642):475-8.

54. Haussmann IU, Bodi Z, Sanchez-Moran E, Mongan NP, Archer N, Fray RG, et al. m(6)A potentiates Sxl alternative pre-mRNA splicing for robust Drosophila sex determination. Nature. 2016:540(7632):301-4.

55. Lence T, Akhtar J, Bayer M, Schmid K, Spindler L, Ho CH, et al. m(6)A modulates neuronal functions and sex determination in Drosophila. Nature. 2016;540(7632):242-7.

56. Shen L, Liang Z, Gu X, Chen Y, Teo ZW, Hou X, et al. N(6)-Methyladenosine RNA Modification Regulates Shoot Stem Cell Fate in Arabidopsis. Dev Cell. 2016:38(2):186-200.

57. Zhang C, Chen Y, Sun B, Wang L, Yang Y, Ma D, et al. m(6)A modulates haematopoietic stem and progenitor cell specification. Nature. 2017; 549(7671):273-6.

58. LV J, Zhang Y, Gao S, Zhang C, Chen Y, Li W, et al. Endothelial-specific $\mathrm{m}(6) \mathrm{A}$ modulates mouse hematopoietic stem and progenitor cell development via Notch signaling. Cell Res. 2018;28(2):249-52.

59. Xu K, Yang Y, Feng GH, Sun BF, Chen JQ, Li YF, et al. Mettl3-mediated m(6)A regulates spermatogonial differentiation and meiosis initiation. Cell Res. 2017;27(9):1100-14.

60. Lin Z, Hsu PJ, Xing X, Fang J, Lu Z, Zou Q, et al. Mettl3-/Mettl14-mediated mRNA N(6)-methyladenosine modulates murine spermatogenesis. Cell Res. 2017;27(10):1216-30.

61. Wojtas MN, Pandey RR, Mendel M, Homolka D, Sachidanandam R, Pillai RS. Regulation of $m(6) A$ Transcripts by the $3^{\prime} \rightarrow 5^{\prime}$ RNA Helicase YTHDC2 is Essential for a Successful Meiotic Program in the Mammalian Germline. Mol Cell. 2017;68(2):374-87.e12

62. Chang $M, L v H$, Zhang W, Ma C, He X, Zhao S, et al. Region-specific RNA $\mathrm{m}(6) \mathrm{A}$ methylation represents a new layer of control in the gene regulatory network in the mouse brain. Open Biol. 2017;7(9): 170166.

63. Wang CX, Cui GS, Liu X, Xu K, Wang M, Zhang XX, et al. METTL3-mediated m6A modification is required for cerebellar development. PLoS Biol. 2018; 16(6):e2004880

64. Ma C, Chang M, Lv H, Zhang ZW, Zhang W, He X, et al. RNA m(6)A methylation participates in regulation of postnatal development of the mouse cerebellum. Genome Biol. 2018;19(1):68.

65. Yoon KJ, Ringeling FR, Vissers C, Jacob F, Pokrass M, Jimenez-Cyrus D, et al. Temporal Control of Mammalian Cortical Neurogenesis by m(6)A Methylation. Cell. 2017;171(4):877-89.e17.

66. Li M, Zhao X, Wang W, Shi H, Pan Q, Lu Z, et al. Ythdf2-mediated m(6)A mRNA clearance modulates neural development in mice. Genome Biol. 2018;19(1):69

67. Engel $\mathrm{M}$, Chen $\mathrm{A}$. The emerging role of mRNA methylation in normal and pathological behavior. Genes Brain Behav. 2018;17(3):e12428.

68. Meyer KD, Saletore Y, Zumbo P, Elemento O, Mason CE, Jaffrey SR. Comprehensive analysis of mRNA methylation reveals enrichment in $3^{\prime}$ UTRs and near stop codons. Cell. 2012:149(7):1635-46.

69. Walters BJ, Mercaldo V, Gillon CJ, Yip M, Neve RL, Boyce FM, et al. The Role of The RNA Demethylase FTO (Fat Mass and Obesity-Associated) and mRNA Methylation in Hippocampal Memory Formation. Neuropsychopharmacology. 2017;42(7):1502-10.

70. Schwartz S, Mumbach MR, Jovanovic M, Wang T, Maciag K, Bushkin GG, et al. Perturbation of m6A writers reveals two distinct classes of mRNA methylation at internal and 5' sites. Cell Rep. 2014;8(1):284-96.

71. Engel M, Eggert C, Kaplick PM, Eder M, Röh S, Tietze L, et al. The Role of m(6)A/m-RNA Methylation in Stress Response Regulation. Neuron. 2018; 99(2):389-403.e9.

72. Nainar S, Marshall PR, Tyler CR, Spitale RC, Bredy TW. Evolving insights into RNA modifications and their functional diversity in the brain. Nat Neurosci. 2016:19(10):1292-8.

73. Li HB, Tong J, Zhu S, Batista PJ, Duffy EE, Zhao J, et al. m(6)A mRNA methylation controls T cell homeostasis by targeting the IL-7/STAT5/SOCS pathways. Nature. 2017;548(7667):338-42. 
74. Yu J, Li Y, Wang T, Zhong X. Modification of N6-methyladenosine RNA methylation on heat shock protein expression. PLoS One. 2018;13(6): e0198604.

75. Su R, Dong L, Li C, Nachtergaele S, Wunderlich M, Qing Y, et al. R-2HG Exhibits Anti-tumor Activity by Targeting FTO/m(6)A/MYC/CEBPA Signaling. Cell. 2018;172(1-2):90-105.e23.

76. Li A, Chen YS, Ping XL, Yang X, Xiao W, Yang Y, et al. Cytoplasmic m(6)A reader YTHDF3 promotes mRNA translation. Cell Res. 2017:27(3):444-7.

77. Li F, Zhang C, Zhang G. m6A RNA Methylation Controls Proliferation of Human Glioma Cells by Influencing Cell Apoptosis. Cytogenet Genome Res. 2019;159(3):119-25.

78. Cui Q, Shi H, Ye P, Li L, Qu Q, Sun G, et al. m(6)A RNA Methylation Regulates the Self-Renewal and Tumorigenesis of Glioblastoma Stem Cells. Cell Rep. 2017;18(11):2622-34.

79. Suvà ML, Rheinbay E, Gillespie SM, Patel AP, Wakimoto H, Rabkin SD, et al. Reconstructing and reprogramming the tumor-propagating potential of glioblastoma stem-like cells. Cell. 2014;157(3):580-94.

80. Xi Z, Xue Y, Zheng J, Liu X, Ma J, Liu Y. WTAP Expression Predicts Poor Prognosis in Malignant Glioma Patients. J Mol Neurosci. 2016;60(2):131-6.

81. Jin DI, Lee SW, Han ME, Kim HJ, Seo SA, Hur GY, et al. Expression and roles of Wilms' tumor 1-associating protein in glioblastoma. Cancer Sci. 2012; 103(12):2102-9.

82. Visvanathan A, Patil V, Arora A, Hegde AS, Arivazhagan A, Santosh V, et al. Essential role of METTL3-mediated m(6)A modification in glioma stem-like cells maintenance and radioresistance. Oncogene. 2018;37(4):522-33.

83. Galardi S, Michienzi A, Ciafrè SA. Insights into the Regulatory Role of m(6)A Epitranscriptome in Glioblastoma. Int J Mol Sci. 2020;21(8): 2816.

84. Lee E, Yong RL, Paddison P, Zhu J. Comparison of glioblastoma (GBM) molecular classification methods. Sem Cancer Biol. 2018;53:201-11.

85. Deng X, Su R, Weng H, Huang H, Li Z, Chen J. RNA N(6)-methyladenosine modification in cancers: current status and perspectives. Cell Res. 2018;28(5): 507-17.

86. Chow RD, Guzman CD, Wang G, Schmidt F, Youngblood MW, Ye L, et al. AAV-mediated direct in vivo CRISPR screen identifies functional suppressors in glioblastoma. Nat Neurosci. 2017;20(10):1329-41.

87. Xi Z, Wang P, Xue Y, Shang C, Liu X, Ma J, et al. Overexpression of miR-29a reduces the oncogenic properties of glioblastoma stem cells by downregulating Quaking gene isoform 6. Oncotarget. 2017:8(15):24949-63.

88. Li F, Yi Y, Miao Y, Long W, Long T, Chen S, et al. N(6)-Methyladenosine Modulates Nonsense-Mediated mRNA Decay in Human Glioblastoma. Cancer Res. 2019;79(22):5785-98.

89. Visvanathan A, Patil V, Abdulla S, Hoheisel JD, Somasundaram K. N ${ }^{6}$ Methyladenosine Landscape of Glioma Stem-Like Cells: METTL3 Is Essentia for the Expression of Actively Transcribed Genes and Sustenance of the Oncogenic Signaling. Genes. 2019;10(2):141.

90. Zhang S, Zhao BS, Zhou A, Lin K, Zheng S, Lu Z, et al. m(6)A Demethylase ALKBH5 Maintains Tumorigenicity of Glioblastoma Stem-like Cells by Sustaining FOXM1 Expression and Cell Proliferation Program. Cancer Cell. 2017;31(4):591-606.e6.

91. Huang $H$, Weng $H$, Deng $X$, Chen J. RNA modifications in cancer: Functions, mechanisms, and therapeutic implications. Ann Rev Cancer. 2019:4:221-40.

92. Wang RJ, Li JW, Bao BH, Wu HC, Du ZH, Su JL, et al. MicroRNA-873 (miRNA873) inhibits glioblastoma tumorigenesis and metastasis by suppressing the expression of IGF2BP1. J Biol Chem. 2015;290(14):8938-48.

93. Luo Y, Sun R, Zhang J, Sun T, Liu X, Yang B. miR-506 inhibits the proliferation and invasion by targeting IGF2BP1 in glioblastoma. Am J Transl Res. 2015;7(10):2007-14.

94. Mu Q, Wang L, Yu F, Gao H, Lei T, Li P, et al. Imp2 regulates GBM progression by activating IGF2/PI3K/Akt pathway. Cancer Biol Ther. 2015; 16(4):623-33.

95. Degrauwe N, Schlumpf TB, Janiszewska M, Martin P, Cauderay A, Provero P, et al. The RNA Binding Protein IMP2 Preserves Glioblastoma Stem Cells by Preventing let-7 Target Gene Silencing. Cell Rep. 2016;15(8):1634-47.

96. Mineo M, Ricklefs F, Rooj AK, Lyons SM, Ivanov P, Ansari Kl, et al. The Long Non-coding RNA HIF1A-AS2 Facilitates the Maintenance of Mesenchymal Glioblastoma Stem-like Cells in Hypoxic Niches. Cell Rep. 2016;15(11):2500-9.

97. Janiszewska M, Suvà ML, Riggi N, Houtkooper RH, Auwerx J, ClémentSchatlo $\mathrm{V}$, et al. Imp2 controls oxidative phosphorylation and is crucia for preserving glioblastoma cancer stem cells. Genes Dev. 2012;26(17): 1926-44.
98. Suvasini R, Shruti B, Thota B, Shinde SV, Friedmann-Morvinski D, Nawaz Z, et al. Insulin growth factor-2 binding protein 3 (IGF2BP3) is a glioblastomaspecific marker that activates phosphatidylinositol 3-kinase/mitogenactivated protein kinase (PI3K/MAPK) pathways by modulating IGF-2. J Biol Chem. 2011;286(29):25882-90.

99. Kleinschmidt-DeMasters BK, Donson AM, Vogel H, Foreman NK. Pilomyxoid Astrocytoma (PMA) Shows Significant Differences in Gene Expression vs. Pilocytic Astrocytoma (PA) and Variable Tendency Toward Maturation to PA. Brain Pathol (Zurich, Switzerland). 2015;25(4):429-40.

100. Bhargava S, Patil V, Shah RA, Somasundaram K. IGF2 mRNA binding protein 3 (IMP3) mediated regulation of transcriptome and translatome in glioma cells. Cancer Biol Ther. 2018;19(1):42-52.

101. Wu C, Ma H, Qi G, Chen F, Chu J. Insulin-like growth factor II mRNA-binding protein 3 promotes cell proliferation, migration and invasion in human glioblastoma. Onco Targets Ther. 2019;12:3661-70.

102. Bhargava S, Visvanathan A, Patil V, Kumar A, Kesari S, Das S, et al. IGF2 mRNA binding protein 3 (IMP3) promotes glioma cell migration by enhancing the translation of RELA/p65. Oncotarget. 2017:8(25):40469-85.

103. Fang $X$, Yoon JG, Li L, Tsai YS, Zheng S, Hood L, et al. Landscape of the SOX2 protein-protein interactome. Proteomics. 2011;11(5):921-34.

104. Golan-Gerstl R, Cohen M, Shilo A, Suh SS, Bakàcs A, Coppola L, et al. Splicing factor hnRNP A2/B1 regulates tumor suppressor gene splicing and is an oncogenic driver in glioblastoma. Cancer Res. 2011;71(13):4464-72.

105. Deng J, Chen S, Wang F, Zhao H, Xie Z, Xu Z, et al. Effects of hnRNP A2/B1 Knockdown on Inhibition of Glioblastoma Cell Invasion, Growth and Survival. Mol Neurobiol. 2016;53(2):1132-44.

106. Gruber AJ, Schmidt R, Ghosh S, Martin G, Gruber AR, van Nimwegen E, et al. Discovery of physiological and cancer-related regulators of 3' UTR processing with KAPAC. Genome Biol. 2018;19(1):44.

107. Park YM, Hwang SJ, Masuda K, Choi KM, Jeong MR, Nam DH, et al. Heterogeneous nuclear ribonucleoprotein C1/C2 controls the metastatic potential of glioblastoma by regulating PDCD4. Mol Cell Biol. 2012;32(20): 4237-44.

108. Klughammer J, Kiesel B, Roetzer T, Fortelny N, Nemc A, Nenning KH, et al. The DNA methylation landscape of glioblastoma disease progression shows extensive heterogeneity in time and space. Nat Med. 2018;24(10):1611-24.

109. Hyman G, Manglik V, Rousch JM, Verma M, Kinkebiel D, Banerjee HN. Epigenetic approaches in glioblastoma multiforme and their implication in screening and diagnosis. Clifton NJ). 2015;1238:511-21.

110. Etcheverry A, Aubry M, de Tayrac M, Vauleon E, Boniface R, Guenot F, et al. DNA methylation in glioblastoma: impact on gene expression and clinical outcome. BMC Genomics. 2010;11:701.

111. Jia $D$, Lin $W$, Tang $H$, Cheng $Y, X u K$, He $Y$, et al. Integrative analysis of DNA methylation and gene expression to identify key epigenetic genes in glioblastoma. Aging. 2019;11(15):5579-92.

112. Hegi ME, Diserens AC, Gorlia T, Hamou MF, de Tribolet N, Weller M, et al. MGMT gene silencing and benefit from temozolomide in glioblastoma. N Engl J Med. 2005;352(10):997-1003

113. Brandes AA, Franceschi E, Paccapelo A, Tallini G, De Biase D, Ghimenton C, et al. Role of MGMT Methylation Status at Time of Diagnosis and Recurrence for Patients with Glioblastoma: Clinical Implications. Oncologist. 2017;22(4):432-7.

114. Ge L, Zhang N, Chen Z, Song J, Wu Y, Li Z, et al. Level of N6Methyladenosine in Peripheral Blood RNA: A Novel Predictive Biomarker for Gastric Cancer. Clin Chem. 2020;66(2):342-51.

115. Konno M, Koseki J, Asai A, Yamagata A, Shimamura T, Motooka D, et al. Distinct methylation levels of mature microRNAs in gastrointestinal cancers. Nat Commun. 2019;10(1):3888

116. Strick A, von Hagen F, Gundert L, Klümper N, Tolkach Y, Schmidt D, et al. The $N(6)$-methyladenosine $(\mathrm{m}(6) \mathrm{A})$ erasers alkylation repair homologue 5 (ALKBH5) and fat mass and obesity-associated protein (FTO) are prognostic biomarkers in patients with clear cell renal carcinoma. BJU Int. 2020;125(4): 617-24.

117. Chai RC, Wu F, Wang QX, Zhang S, Zhang KN, Liu YQ, et al. m(6)A RNA methylation regulators contribute to malignant progression and have clinical prognostic impact in gliomas. Aging. 2019;11(4):1204-25.

118. Cheng Y, He C, Wang M, Ma X, Mo F, Yang S, et al. Targeting epigenetic regulators for cancer therapy: mechanisms and advances in clinical trials. Signal Transduct Target Ther. 2019;4:62.

119. Senft D, Oi J, Ronai ZA. Ubiquitin ligases in oncogenic transformation and cancer therapy. Nat Rev Cancer. 2018;18(2):69-88. 
120. Cully M. Chemical inhibitors make their RNA epigenetic mark. Nat Rev Drug Discov. 2019;18(12):892-4.

121. Selberg S, Blokhina D, Aatonen M, Koivisto P, Siltanen A, Mervaala E, et al. Discovery of Small Molecules that Activate RNA Methylation through Cooperative Binding to the METTL3-14-WTAP Complex Active Site. Cell Rep. 2019;26(13):3762-71.e5.

122. Tzelepis K, De Braekeleer E, Yankova E, Rak J, Aspris D, Domingues AF, et al. Pharmacological inhibition of the RNA $\mathrm{m}^{6} \mathrm{~A}$ writer METTL3 as a novel therapeutic strategy for acute myeloid leukemia. Blood. 2019;134:403.

123. Wu R, Li A, Sun B, Sun JG, Zhang J, Zhang T, et al. A novel m(6)A reader Prrc2a controls oligodendroglial specification and myelination. Cell Res. 2019;29(1):23-41.

\section{Publisher's Note}

Springer Nature remains neutral with regard to jurisdictional claims in published maps and institutional affiliations.

Ready to submit your research? Choose BMC and benefit from:

- fast, convenient online submission

- thorough peer review by experienced researchers in your field

- rapid publication on acceptance

- support for research data, including large and complex data types

- gold Open Access which fosters wider collaboration and increased citations

- maximum visibility for your research: over $100 \mathrm{M}$ website views per year

At BMC, research is always in progress.

Learn more biomedcentral.com/submissions 